Dr. Tregear has tried to bring together the basic data necessary for the use of planners and administrators, and to indicate the sources from which fuller information can be obtained. His is a factual survey, a record of things as they are, not a forecast of possible developments nor a catalogue of suggested remedies for the territory's land-use problems. This is exactly as it should be and it is indeed the justi fication for all land-use surveys. They may be difficult to carry out and they may sometimes seem very wasteful of time and energy; yet without them there can be no precise and objective assessment of land use as it can be observed to-day. Subjective judgments, wishful thinking and intelligent anticipation are all specifically excluded from the purpose of the World Land Use Survey.

In essence, Dr. Tregear's monograph is an exposition and elaboration of the land-use map prepared. At times the literary style is weak, and occasionally the text becomes little more than a series of notes. A few of the diagrams are disappointing because they cannot be read easily or have been excessively reduced. Sometimes Dr. Tregear might have emphasized more those relationships of physical and human phenomena that geographers claim as a special part of their field. There may well be mono- graphs with a rather more penetrating analysis later in this series. Nevertheless, the volume deserves a welcome by geographers and others both for its own worth and as an indication of what may be expected for other areas in the future. It is a sign that the World Land Use Survey has passed beyond the talking and planning stages and, in some countries at least, beyond the field-work to the point where maps and reports are being produced. Clearly the editor, Prof. Stamp, who has just retired from his chair in the University of London, is going to be as active as ever in the years to come. We can, therefore, anticipate a series of regional monographs for the world that will be comparable in conception, content and importance to the well-known County Reports of the Land Utilisation Survey of Britain. ROBERT W. STEEL \footnotetext{
Rawson, R. R., and Sealy, K. R., "Land Use Map of Cyprus",
35 in. $\times 22$ in. (Bude: Geographical Publications, Ltd., 1956.) $3 s$. 2 Cole, M. M., "Land Use Studies in the Transvaal Lowland" (World Land Use Survey : Occasional Paper 1). Pp. $41+37$ figs. (Bude : Geographical Publications, Ltd., 1956.) 5s.

${ }^{3}$ Tregear, T. R., "A Survey of Land Use in Hong Kong and the New Territories"'. (The World Land Use Survey : Monograph 1.) Pp. $v+75+1$ map. (Hong Kong: Hong Kong University Press Bude : Geographical Publications, Ltd., 1958. Distributed outsíde Hong Kong by Macmillan and Co., Ltd.) 12s. 6d.
}

\title{
THE FRESHWATER BIOLOGICAL ASSOCIATION
}

$\mathrm{B}_{\mathrm{B}, \mathrm{i}}$ ESIDES details of the administration, organization and state of the finances of the Freshwater Biological Association for 1957-58, the twenty-sixth annual report contains a statement by the Director on the scientific work of the staff.

In the chemistry section analyses of algae have shown that cobalt, copper and zinc are present in the plant tissues in amounts the ratios of which are similar to those of their concentrations in the environment, a fact which suggests that the uptake of such elements by algae may be governed rather by their concentrations in the surrounding water than by any physiological needs of the plant.

Earlier work on the acid waters of blanket bogs in the Pennines and of bog lakes in Nova Scotia suggested that both owed their acidity to sulphuric acid. Dr. E. Graham has now extended these studies to bog waters elsewhere in Britain and in less-oceanic sites in Poland with similar results. Since sulphuric acid also seems to cause the acidity of the soil solutions extracted from heavily leached mor humus layers overlying podzolic soils in Lake District oak woods, it is probably to be regarded as an important agent in soil leaching. While in Britain it comes largely from atmospheric pollution brought down in rain, there is evidence that it is also produced by the breakdown of organic sulphur compounds in the soil ; it may therefore be important in unpolluted areas also.

Continuing his observations on the fluctuations in numbers of the smaller nannoplankton, Dr. J. W. G. Lund finds a clear difference between the periodicity of the very small green algae in the surface waters of Windermere, Esthwaite Water and Blelham Tarn and that of the larger species. Thus, large populations of the small forms have been observed in late winter and early spring. It will, however, be necessary to continue observations over several years to determine whether this happens regularly.
Work on the polycentric chytrids which occur in the reed beds of Windermere has been continued by Dr. L. G. Willoughby, who is making a comparative study of eight isolates of Cladochytrium replicatum. So far, particular attention has been paid to growthrates and fruiting (that is, zoosporangium formation) on agar. One of the isolates has been uniformly sterile under all conditions, while another has only rarely formed zoosporangia. Two further isolates of the eight can be segregated not only on purely morphological grounds, but also because the rate of linear growth on agar is consistently high. The effect of light on growth and fruiting in this fungus has been investigated. Light is not essential for pigment and hence zoosporangium formation, and total growth, as measured by the increase in colony diameter on agar, is independent of this factor. It seems clear, however, that fruiting is generally greater in light than in the dark, the effect being particularly noticeable in the isolates with high fruiting capacities.

A further study has been made of the influence of the container on the rate at which bacteria multiply in samples of lake water stored in the laboratory. This has been extended to cover polyethylene and nylon containers as well as those of silica, 'Pyrex' and soda-glass. The conclusion reached previously that silica provided the least 'active' surface for bacterial growth was confirmed; the others follow in the order 'Pyrex', soda-glass, polyethylene up to nylon, which stimulates the largest bacterial growths. It appears that the surface properties of polyethylene and nylon in particular facilitate the supply of something the bacteria need, whereas silica has no such property, regardless of the type of water, the temperature, or of whether the containers are stationary or shaken. This is borne out by the discovery that in experiments using a constant circulation of water, considerable multiplication of bacteria took place wherever the 
liquid remained in contact with polyethylene or nylon connecting tubes. These results may have direct practical importance on industrial process problems, where polyethylene pipes become blocked by masses of slime-forming bacteria attached to the walls, and in the storage of non-sterile solutions in containers made of these materials.

The use of opercular bones to estimate the age and past growth of pike has been further investigated by Dr. Winifred Frost and Miss Charlotte Kipling, who concluded last year that growth can be calculated accurately by simple proportion from measurements of the annual rings, making an arbitrary choice by inspection of a centre from which to make the measurements. Since the rings near the centre are not always clearly visible, the method does not give reliable estimates of growth in the early years of life. This difficulty has been overcome by the use of theoretical growth-tables derived from Ford's growth formula, using data from Windermere calculated for slow-, medium-, fast- and very fast-growing fish for the two sexes separately. By comparing the growth in later years of any individual fish with these tables, it is usually possible to assign it to one of the growthrate categories. This makes it possible to infer its true age and so to decide whether the first discernible ring represents its first, second or third winter.

D. F. Westlake has measured the rate of production of oxygen by Ranunculus spp. in a closed, static system, at various light intensities. The highest gross rate of oxygen production found for lightsaturated plants was about $10 \mathrm{mgm}$. of oxygen $\mathrm{hr}$./gm. (dry weight) at $15^{\circ} \mathrm{C}$. Light-saturation was approached at 10,000 lux, and both this and the maximum rate of oxygen production are lower than generally reported in the literature on higher plants. Possibly this is the result of using static conditions for the experiments, and apparatus for determining oxygen production in flowing conditions is being developed.

\section{MECHANICAL ENGINEERING RESEARCH LABORATORY}

\section{REPORT FOR 1957}

$\mathrm{T}$ WE report on the work of the Mechanical Engineering Research Laboratory for $1957^{*}$ makes fascinating reading, and contains much that will interest any engineer and many physicists.

The chairman of the Engineering Research Board directs attention in his report to the difficulty of getting the results of the Laboratory's work both disseminated and applied. Most engineering scientists have yet to learn that a paper which may lead in due course to scientific honour is unlikely to be understood by many practising engineers, however high their theoretical attainments; the work must also be published as a straightforward physical description, written in lucid English and devoid of mathematics if it is to be read and applied. Sir Charles Inglis used to state this truth regularly in his lectures; few have learned the lesson.

The proportion of scientific and experimental staff is 160 out of a total of 444 . This seems high. Does the Laboratory suffer from the difficulty common in

* Department of Scientific and Iudustrial Research. Mechanical Engineering Research 1957: Report of the Mechanical Engineering Research Board, with the Report of the Director of Mechanical Engineering Research. Pp. iv $+63+4$ plates. (London: H.M. Stationery Ottice, 1958.) 4s. $6 d$. net.
Government and university laboratories of being hamstrung financially in providing adequate ancillary and office staff?

In recommending a rapid expansion of the Laboratory, the problem of how it, the universities and the colleges of advanced technology are all to be staffed must arise. In Germany, most of the work similar to that done at the Mechanical Engineering Research Laboratory is done in institutes of the Technische Hochschulen. Might it not be better to give the colleges of advanced technology similar institutes?

While it is clear why some of the research, both fundamental and applied, is being done nationally, it is surprising to find that fundamental work on greases has not already been undertaken on an adequate scale by private industry. When such gaps are found, is it not desirable that they should be investigated where the staff concerned can also be available for part-time teaching ?

Nevertheless, the report describes the development of fine facilities and the accomplishment of much excellent work. Many firms should read it and send members of their staff to see what is being done.
D. B. WELBOURN

\section{TILT CRITERIA AND DIRECTION OF ROTATION OF SPIRAL GALAXIES}

G DE VAUCOULEURS has a paper with the J. above title in the Astrophysical Journal, 127, 2 ; March 1958, in which he presents a review of the observational evidence on the direction of rotation of the spiral pattern in ordinary and barred spirals. A growing body of evidence suggests that the formation of spiral structure is governed by the large-scale magnetic and hydrodynamical properties of the interstellar medium in rotating galaxies, and according to current ideas, dust or 'smoke' particles may form out of the gaseous medium and induce the condensation of gas clouds into stars or groups of stars. The author of the paper has discussed the morphology of spiral structure and its bearing on the large-scale instantaneous distribution of gas and dust in galaxies (Hand. Phys., 53), and in the present paper he deals with the systematic motion of the interstellar substratum on the largest scale in a galaxy, in other words, with the general rotation of the spiral pattern. The previous investigations of those who have devoted their attention to this subject-Baade, Hubble, Lindblad, Stebbins, Zwicky and others-are referted to, and the reasons for the divergences in the interpretations are analysed. The discussion is recapitulated in Table 1, p. 499, which consists of eleven columns (the symbols used in these are explained at the end of the table and also on the previous page) and the conclusions are briefly as follows. 